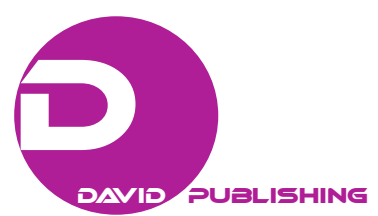

\title{
Healthcare Application: An Analysis of Reception Studies in Interactive Digital Television
}

\author{
Alexandre Kieling, Amanda Caroline Rodrigues, Élida Borges, Jhéssika Almeida, Watson Odilon \\ Catholic University, Brasília, Brazil \\ Áulus Diniz, Diogo Caetano \\ Brasilia University, Brasília, Brazil \\ Kênia Freitas \\ Communication Paulista State University Julio Mesquita, Bauru, Brazil
}

\begin{abstract}
This paper presents a healthcare application for Brazilian digital television, its main purposes and its reception tests with end users. This is an applied research for testing the scope of the Global ITV project funded by the Brazilian National Council for Scientific and Technological Development (CNPq) and the European community which has developed a hybrid broadband and broadcasting platform.
\end{abstract}

Keywords: interactive digital television, TV application, healthcare, Ginga ecosystem, Global ITV project

\section{Introduction}

While the television technology was born as unidirectional medium for audiovisual transmission, the latest years have redefined its functionalities and uses, following the signal transition from analog to digital and the arrival of the Internet to TV screens. In the end of the 20th century, theorists debated the passage from paleotelevision to neotelevision (Eco, 1986; Casseti \& Odin, 1990), but now both terms have been superseded by a new television era, which combines digital technology, synchronism with other media and new formats and uses: the hypertelevision (Scolari, 2008).

Scolari (2008) proposed that this transformation is not only technological but also social, as spectators transfer their fruition experiences from other media to this new television. This is a hypertextual fruition experience, whose spectators become used to interactivity and networking, experting in fragmented textuality, and is highly capable of adapting to new interaction media. This is the context digital television needs to adapt to.

According to Orozco (2010), this phenomenon created a new relationship between the audience and

Alexandre Kieling, Ph.D. in Communication, Stricto Sensu Program in Creative Economy, Catholic University of Brasilia (UCB), Brasilia, Brazil

Amanda Caroline Rodrigues, Master in Communication, Catholic University of Brasilia (UCB), Brasilia, Brazil.

Élida Borges, Master in Communication, Catholic University of Brasilia (UCB), Brasilia, Brazil.

Jhéssika Almeida, Journalism Student, Catholic University of Brasilia (UCB), Brasilia, Brazil.

Watson Odilon, Ph.D. in Communication, Catholic University of Brasilia (UCB), Brasilia, Brazil.

Áulus Diniz, Engineering Student, Brasilia University (UnB), Brasília, Brazil.

Diogo Caetano, Ph.D. in Engineering, Brasilia University (UnB), Brasília, Brazil.

Kênia Freitas, Ph.D. in Communication, Researcher, Communication Paulista State University Julio Mesquita, Bauru, Brazil. 
multiple screens, given new interactivity, convergence, and individualization capabilities in the media. Instead of spectators, there are now users, who dissolve classical styles of consumption and interaction with information and images. They are also viewers/internauts who insert themselves in the regular processes of production, circulation, and reception, due to the profusion of digital devices (Kieling, 2009). The processes of recognition and circulation, according to Verón's (2004) social semiosis, are under intense and multidirectional flow. The television in the living room is not alone anymore, and the viewers do not immerse themselves in audiovisual content only as groups, seating down on armchairs and sofas.

Audiovisual mass consumption is currently under a paradigm dispute between fixed-grid and on-demand models. Furthermore, in Latin-American countries, such as Brazil, the definitive migration from the analog to the digital signal is still under course. Viewers, users, producers, and programmers are still discovering and building new possibilities, functionalities, and uses for this hypertelevision. Under this context of experimenting and experiencing, this paper presents a healthcare-related application for Brazilian digital television.

The application was developed in 2015 by a team at Brasília's Catholic University (UCB), under the scope of a larger project called Global ITV ${ }^{1}$ (http://www.globalitv.eu), which developed an interoperable converter prototype, suited for the Brazillian/Latin-American and European television systems. A healthcare-related application was developed for the Brazilian platform, $\mathrm{Ginga}^{2}$, as a part of the functionality tests for Global ITV's converter. The application presents standard health information for the average Brazilian user, such as symptoms and prevention measures for chronic diseases, like diabetes and hypertension, as well as the corresponding services and treatments available at the national public system (SUS), such as free medication and exams.

Such an application is justified by the difficult access to this kind of information by the standard Brazilian/Latin-American citizen, whose Internet access is still scarce. Full Internet connection is currently a developed-world privilege, and seemingly will continue to be for a long time. In this context, the application's main objective was to offer a simple and intuitive interface, allowing access to Brazilian health-related information through the Brazilian television terrestrial transmission and its middleware, Ginga. This pilot application was developed such that it can be easily extended by adding other diseases and information. The application's test cases also hint at some of the utilization and reception capabilities by the end users.

\section{Tests Description and Methodology}

The Healthcare ITV application presents the main benefits offered by the Brazilian Public Health System, organized by diseases. The proposed application is not only of public interest, but it also fits in a wide variety of TV programmes, such as public-TV coverage of SUS and of specific diseases, as well as public healthy-lifestyle promotion.

The first diseases considered are those with the highest death rates in the country, such as diabetes mellitus,

\footnotetext{
1 The GLOBAL ITV project was contemplated in the 2nd Brazil-EU Call No. 13/2012 of the National Council for Scientific and Technological Development $(\mathrm{CNPq})$, which is part of the Brazil-European Union Cooperation Program in partnership with the Seventh Framework Program (FP7). Titled Global ITV: Interoperability of Interactive and Hybrid TV Systems. The Brazilian consortium led by the University of São Paulo (USP) under the coordination of professors Marcelo Knörich Zuffo, PhD and. Moacyr Martucci PhD - of which the Catholic University of Brasilia is part.

2. Middleware adopted in Brazil and other 13 Latin-American countries. The Brazilian terrestrial transmission system results from a combination of the Japanese audiovisual transmission system, IDB-T, and a Brazilian data transmission system called Ginga, developed by Brazilian researchers led by Rio de Janeiro's Catholic University.
} 
hypertension, and cancer. Of course, mapping all of this information is not only a gargantuan task, but it also falls out of the scope of the Global ITV project. In this manner, we propose to tackle the information regarding diabetes mellitus, which is of great health concern and specific enough to make the job feasible, given the time restraints of the Global ITV project.

Even though digital TV technology is not suited for applications that access great databases and individual content presentation, it is possible to overcome these limitations through Internet access to data servers and data filtering. For instance, a hybrid digital TV application can consult large databases through communication services between applications called web services.

Subjective tests in Europe were organized by A-CING and carried out between the 26th and 27th of October 2015 in the facilities of Cellnex-former Abertis Telecom-in Tres Cantos, Madrid, Spain ${ }^{3}$. As previously planned, after an initial explanatory talk, for each participant, the test was divided in two parts. In the first part, the participant had to test the two Global ITV applications developed (healthcare application and social messaging application) together with a third existing commercial application by carrying out certain tasks assigned. In this article, we will focus on the results of the healthcare application.

A-CING's subjective tests for the applications developed by the Global ITV team were carried out in a controlled lab environment at Cellnex Laboratories, in Tres Cantos, Madrid, Spain. Ten individuals participated in this experiment as users. The overall test was divided into three stages:

Stage 1: Initial welcome, explanatory talk for the participants and forms filling;

Stage 2: Individual test of the applications;

Stage 3: An individual usability test in relation to their experience using the applications.

For this test, the chosen methodology for measuring usability and user experience was the System Usability Scale questionnaire. This is a simple and widely used method which has proven reliable results with sample sizes similar to the one available for this environment. It is a 10-item scale questionnaire (Likert scale) based on forced-choice questions, where a statement is made and the respondent then indicates the degree of agreement or disagreement with the statement on a 5-point scale.

One of the key aspects for obtaining reliable results with a low number of participants is that System Usability Scale questions all address different aspects of the user's reaction to the application as a whole (e.g., "I found the application unnecessarily complex" and "I felt very confident using the application") as opposed to asking the user to assess specific features of the applications (visual appearance, organization of information, etc.).

The questionnaire is formed by the following 10 items:

(1) I think that I would like to use this application frequently;

(2) I found the application unnecessarily complex;

(3) I thought the application was easy to use;

(4) I think that I would need the support of a technical person to be able to use this application;

(5) I found the various functions in this application were well integrated;

(6) I thought there was too much inconsistency in this application;

(7) I would imagine that most people would learn to use this application very quickly;

(8) I found the application very cumbersome to use;

3 The full test report can be consulted at the document "User test results and acceptance report" in: http://www.globalitv.org/Publications/GLOBALITV-D5.5-UserTestResultsAndAcceptanceReport.pdf. 
(9) I felt very confident using the application;

(10) I needed to learn a lot of things before I could get going with this application.

The System Usability Scale questionnaire was given to the respondent after they interacted with the application being evaluated, but before any debriefing or discussing takes place. To follow the protocol, respondents should be asked to record their immediate response to each item, rather than thinking about them for a long time. According to the report of the test, the methodology was straight followed and all items in the questionnaire were checked. And when a respondent feels that they could not respond to a particular item, they were oriented to mark the centre point of the scale.

As outlined in the report, System Usability Scale produces a single number representing a composite measure of the overall usability of each application. Scores for individual items are not meaningful on their own. To calculate the score, it is necessary first to sum the score contributions from each item. Each item's score contribution will range from 0 to 4 . For Items 1, 3, 5, 7, and 9, the score contribution is the scale position minus 1 . For Items $2,4,6,8$, and 10, the contribution is 5 minus the scale position. Then, it is necessary to multiply the sum of the scores by 2.5 to obtain the overall value. Therefore, despite that the usability and user experience score given by the System Usability Scale questionnaire for each participant varies from 0 to 100 , this result does not represent a percentage.

The three stages of the testing have been already described. Now, they are described in detail (always according to the description from the report):

Stage 1. The first part of the experiment consisted on giving and collecting information to and from the participants. For operational reasons, the participants were splitted in two groups of five people. Initially, the group was given an introduction to the Global ITV project, its objectives and impact, and more specifically, the objectives of the subjective tests that they were about to participate. After this, a brief description of the three iTV applications to be tested was provided to the participants, just to let them know what was they about. Finally, a description of the test methodology was given. If users had any question about this it was solved.

After that introduction and following the FP7 recommendation on Ethics for Researchers, each participant was handed a participant form where all details about their rights as participants and personal records management were provided before the testing and after the explanation. Moreover, an individual profile form was filled in by each participant in order to gain some information about their age and gender, level of studies, and usage of technology.

Following the initial stage, the two other stages were carried out individually by each participant.

Stage 2. The second stage was focused on the individual test of three iTV applications. For each application, the participant was asked to carry out two different tasks in order to interact with the application and get a feel of its usability.

Stage 3. Once the testing with Global ITV equipment was ended, each participant had to fill in a usability form in order to assess their subjective impression of the three applications. As System Usability Scale methodology recommends, users had to fill same form for every single app tested. (Miranda et al., 2016)

The first set-up depicts the test environment for the healthcare application designed as an information system for the Brazilian national health system. For this set-up, or either for the other Global ITV application, a television signal was not broadcasted. Instead, an encoded transport stream (TS) with and attached carousel object was stored in a PC and used by a coding and modulation software (Dektec StreamXpress) and hardware (Dektec DTU-215 modulator) to produce the ISDB-T television signal which was directly fed into the Global ITV set-top box (STB). Figures 1 and 2 (extracted from the report) illustrate the operation.

For the test, two tasks were chosen for the participants to perform. These where:

Task 1: Find information about clinic obstetrics.

Task 2: Find information about the symptoms of the diabetes. 


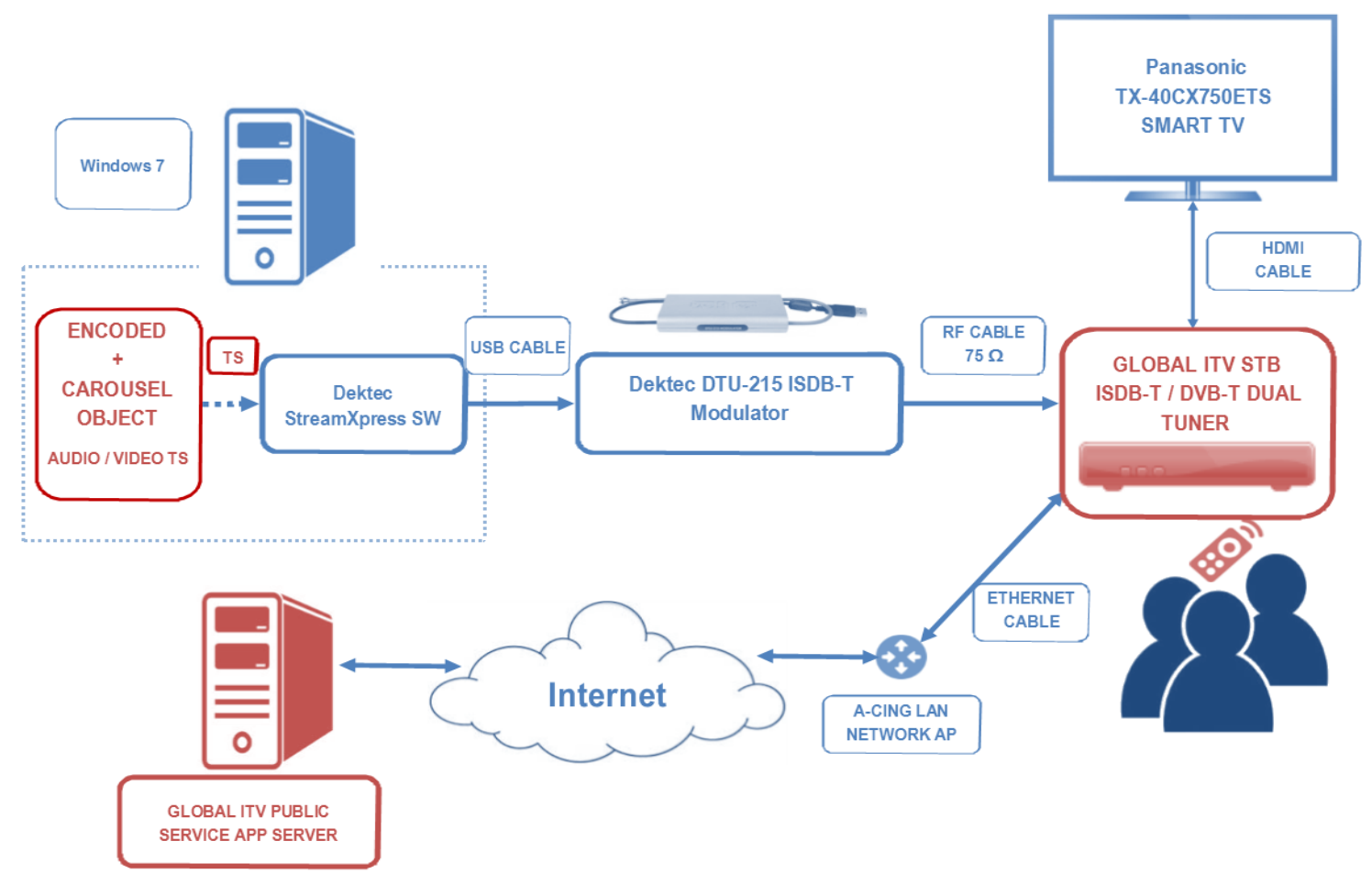

Figure 1. Test set up for Global ITV healthcare application.

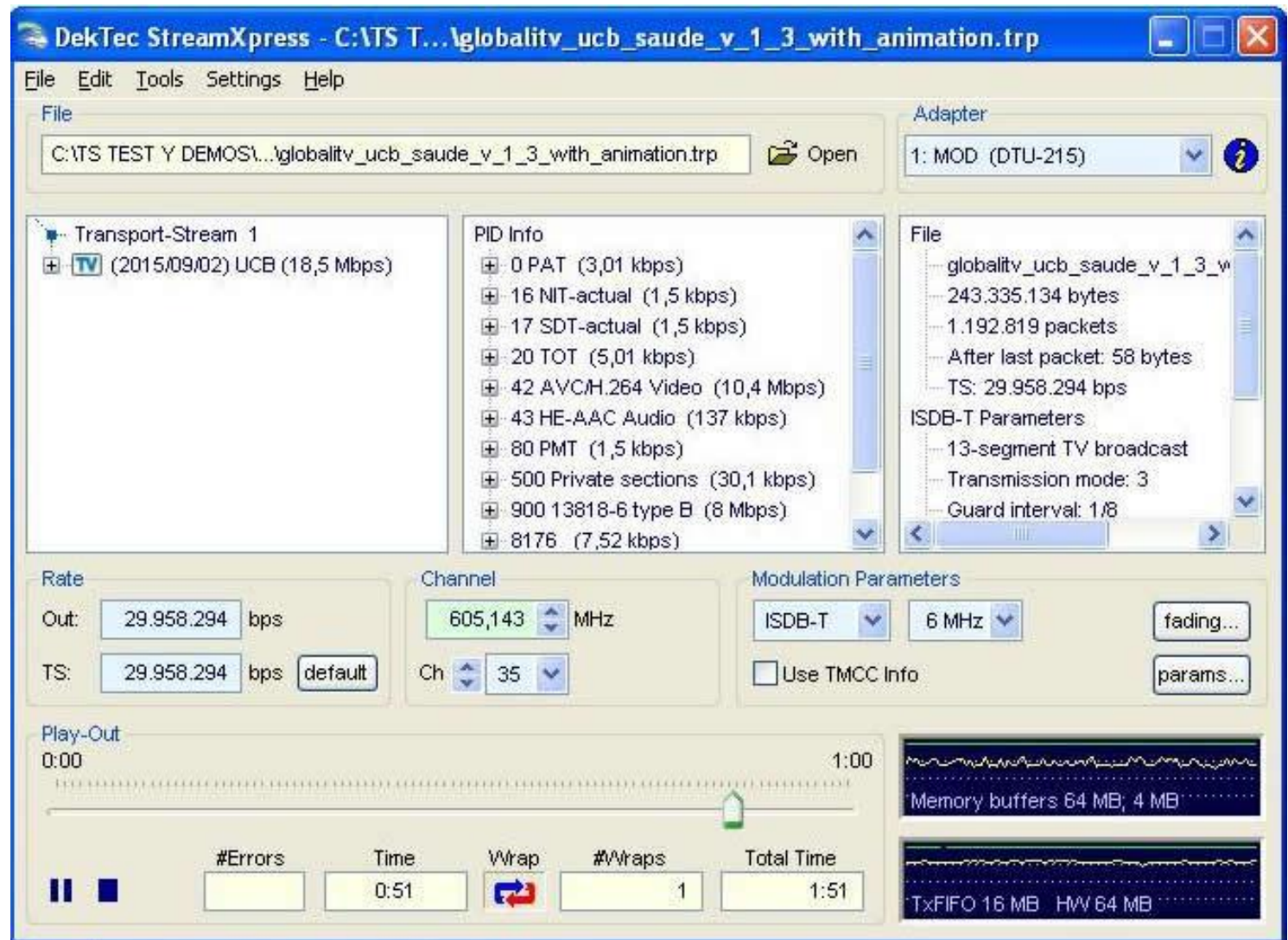

Figure 2. StreamXpress ISDB-T parameters. 
According to the report description, all participants were asked to carry out these tasks without a detailed knowledge of the applications; just a general oral description was given. The objective was that the participants would face the usage of the applications like they would do it for the first time in their houses, getting an initial glance about how intuitive and usable they were. Despite that not counting with assistance or extra information during the tests, a member of the research team always supervised the tests in order to assist the participants with major problems, such as problems with the hardware (television signal, cables, etc.) and software (application glitches).

It is significant to remark that all participants were able to carry the tasks within the six minutes of time they were given.

\section{Results}

According to the report, the main constraint faced for A-CING's subjective tests was the minimum amount of participants available for carrying out the tests, only 10 in the end. From the technical point of view, the Global ITV applications presented small glitches due to their early prototype version (e.g., the STB had to be reset if the Ginga application was closed with the red button since it could not be relaunched) but these did not affect the performance of the tests.

Tables 1 and 2 will briefly present the data collected during the test. The considerations about their meanings will be presented at the conclusion of this article.

\section{System Usability Scale Analysis}

The calculation of the SUS form results for each application and each participant is shown in Table 1 . From them, the average mark for each application is calculated: 70.79 for the Ginga application, 79.80 for the HbbTV application, and 81.13 for the commercial HbbTV RTVE application.

Table 1

System Usability Scale Results for User Tests

\begin{tabular}{lllll}
\hline Participant & SUS App 1: Healthcare & SUS App 2: Social messaging & SUS App 3: RTVE & Best score \\
\hline 1 & 62.50 & 80.00 & 75.00 & HbbTV \\
2 & 82.50 & 77.50 & 80.00 & Ginga \\
3 & 72.50 & 85.00 & 75.00 & HbbTV \\
4 & 60.00 & 72.50 & 77.50 & RTVE \\
5 & 85.00 & 80.00 & 100.00 & RTVE \\
6 & 50.00 & 67.50 & 82.50 & RTVE \\
7 & 80.00 & 70.00 & 67.50 & Ginga \\
8 & 65.00 & 87.50 & 72.50 & HbbTV \\
9 & 75.00 & 97.50 & 97.50 & HbbTV/RTVE \\
10 & 85.00 & 85.00 & 90.00 & RTVE \\
AVG & 70.79 & 79.80 & 81.13 & \\
Classification & Acceptable-Ok grade & Acceptable-Good grade & Acceptable-Good grade & \\
\hline
\end{tabular}

To understand these numbers, it is important to know that: According to the classification given in, products which are at least passable in terms of usability have SUS scores above 70, with better products scoring in the high 70s to upper 80s (although high acceptability in the field is not guaranteed), with truly superior products scoring over 90 . And that products with scores lesser than 70 should be considered candidates 
for increased scrutiny and continued improvement and should be judged to be marginal at best. For scores under 50, it can be almost certain that the produce will have usability problems in the field.

Looking at the scores of the applications in Table 1, it can be seen how the healthcare application barely scores above 70, whereas the social messaging scores about 80 , and the RTVE scores a bit more than 81 . Following the score interpretation guidelines, it can be seen how all of them fell into the acceptance area, but different considerations need to be taken into account for all of them. According to the report, for the healthcare application the result suggests that further development and improvement needs to be performed if at least acceptability in the market is soak, with further improvement for high acceptance.

These results were accompanied by an extra section with open and subjective questions about the applications. For healthcare application, the positive and negative points highlighted by the participants were:

Table 2

Positive and Negative Points Highlighted for Healthcare Application.

\begin{tabular}{|l|l|l|}
\hline Global ITV healthcare application & \multicolumn{2}{|l|}{} \\
\hline Positive remarks & Negative remarks & Recommendations \\
\hline Easy to use & $\begin{array}{l}\text { "Pregnancy" is under the category } \\
\text { "Illness"-N.B. Though this is not true if } \\
\text { we look into the app, the participant } \\
\text { reflected this remark in his/her } \\
\text { comments. } \\
\text { Arrows in green and red icons produce } \\
\text { erors; people look for arrows and not } \\
\text { colours in the remote control. }\end{array}$ & $\begin{array}{l}\text { Images and videos should be added. } \\
\text { Bigger variety in menus. } \\
\text { More attractive menus. } \\
\text { First explanatory layer } \\
\text { Be able to search for terms }\end{array}$ \\
\hline
\end{tabular}

Therefore, according to this extra questionnaire, from the comments on the healthcare application, on the positive side, it was remarked how the application is easy to use for the user since the first moment. However, several users commented on the fact that the icons used for the "Start" and "End" options of the application where confusing since they made them focusing more on the shape of the icon rather than on the colour. And also, several users suggest more attractive menus and the inclusion of multimedia content together with a "Search" option.

\section{Conclusions}

The tests indicate positive user reactions to new reception instances with new technologies exhibiting and propagating audiovisual content. The television is a terrestrial transmission process with technology swinging from Hertzian waves and from cathode rays tubes to binary codes and data processing in conjunction with audio and video. In comparison to devices which the usability and functionality are originally come from a digital culture, theirs adaptation and manipulation by the user go through a mutual learning curve.

From this perspective, it is noteworthy that the relationship with the remote control, the navigation, and the parallel content to television flow shows itself, in a sense, promising. The experiment has generated more interest from the viewers/user, suggesting changes, improvements, and other alternatives. There has not been a negative reaction advising against new experiences or even the suggestion of its absolute cancellation.

The feedback received indicates the need of improvement on the menu bar, the icons and the attractiveness point for a better possibility of consumption. One should not forget the application was tested in a public that understood clearly how interactivity and navigation data occurs. It is thus a sample which separates the viewer 
from the web user. This is a group that is even used to applications developed in HTML5, which is a common language to networks and their devices (computer, tablet, smartphone, and TV).

When this audience reacts positively to an application developed exclusively in the logic of broadcast TV, with its technological evolution curve still in progress, this is an encouraging sign.

At least two trends are observed:

(1) The free broadcast television far from been a disposable content distribution system is a mean that has not yet been exhausted in its technological and economic possibilities;

(2) The renewal of the conditions of production, circulation, and consumption - with TV digitalization-it is a way to ensure access to populations who are still without the diversity of connectivity options. Therefore, it has also a possibility of social and cultural use.

It is a fact that there are indicators of a future trend with centrality of the distribution of content via fixed network infrastructures, and especially via mobile network. It is also a fact that the investments will be concentrated in areas of economic sustainability, as can be seen nowadays in the distribution of global capital. It is hard to imagine that the distribution of content will bypass the neoliberal rationality that organizes individual and planetary priorities. Therefore, It is in this complex scenario that are being established the logic of the viewer, the internaut, and the user. There is a space in which is believed there is reason for the existence of an interoperable platform and for an evolution of the distribution of data and interactivity for terrestrial broadcast TV.

Considering this picture, the experiment of the healthcare application, even in the reflective effort of a brief paper, seems to leave us leads and indications to not turn the page in the studies and experiments with the digital terrestrial interactive TV.

\section{References}

Casetti, F., \& Odin, R. (1990). De la paléo à la néo-télévision (From the paléo to the néo-télévision). Communications, 51(1), 9-26.

Eco, U. (1986). T.V. la transparencia perdida. In La estrategia de la ilusión (TV. The lost transparency. In The Illusion Strategy). Barcelona: Lumen. Retrieved from https://omegalfa.es/downloadfile.php?file=libros/t-v-la-transparencia-perdida.pdf

Kieling, A. S. (2009). Televisão: A presença do telespectador na configuração discursiva da interatividade no programa "Fantástico" (Television: The presence of the viewer in the discursive configuration of interactivity in the program "Fantástico"). Tese de doutorado na Unisinos, São Leopoldo.

Miranda, F. C., Eliasquevici, M. K., Malcher, M. A., Marques, J. A., Saruffo, M., Mancebo, J., ... Globalitv, A. (2016). D5.5: User test results and acceptance report. Internal report, Madrid, Spain.

Orozco, G. (2010). Las Audiencias y patalhas. Lo nuevo e lo viejo y lo que viene (Hearings and screens. The new and the old and what is coming). In C. Guiliana (Ed.), Televisión: 14 formas de mirala. Lima: Departamento Académico de Comunicaciones. Pontificia Universidad Católica del Perú.

Scolari, C. (2008). Hacia la hipertelevisión. Los primeros síntomas de una nueva configuración del dispositivo televisivo (Towards hypertension. The first symptoms of a new configuration of the television device). Retrieved from https://dialnet.unirioja.es/descarga/articulo/2694422.pdf

Verón, E. (2004). Fragmentos de um tecido (Fragments of a fabric) (1st ed.). São Leopoldo: Unisinos. 